\title{
Combination of DOA and beamforming in position-based routing for underlay cognitive wireless networks
}

\author{
L. De Nardis, M.-G. Di Benedetto \\ DIET Department \\ Sapienza University of Rome \\ Rome, Italy \\ Email: \{lucadn, dibenedetto $\} @$ newyork.ing.uniroma1.it
}

\author{
A. Akhtar, O. Holland \\ King's College London \\ London, UK \\ Email: \{oliver.holland, auon.akhtar\}@kcl.ac.uk
}

\begin{abstract}
This work presents a routing strategy for underlay cognitive wireless networks combining a position-based routing protocol, with position information obtained by means of Direction Of Arrival (DOA) estimation, with beamforming. Beamforming takes advantage of the estimated DOAs to maximize Signal-to-Noise Ratio at the intended receiver while minimizing interference towards potential victim receivers, either cognitive or primary. The paper first introduces the location-based routing protocols considered in this work; next, the DOA estimation and beamforming techniques adopted in conjunction with the routing protocol are described. The proposed strategy is then presented and evaluated by means of computer simulations. Simulation results highlight the increase in network performance in terms of end-to-end throughput guaranteed by the proposed strategy, thanks to the reduction of internal interference experienced by cognitive terminals as a result of the introduction of beamforming combined with the decrease in control packets transmissions guaranteed by the use of position information.
\end{abstract}

\section{INTRODUCTION}

Position information can be introduced in almost all aspects of network organization and management, from medium access control to node clustering, from scheduling to routing. Routing, in particular, is a network task that can take advantage of the availability of position information. The introduction of position information in the routing process can lead to significant performance improvement in the specific case of cognitive radio networks. A recent evolution in the field of cognitive radio regards in fact the introduction of external databases capable of providing information about the presence, activity and physical position of primary transmitters, to be accessed by secondary cognitive devices in order to determine spectrum availability by building what is currently referred to as a Radio Environment Map. Although the approach was mainly proposed to regulate and enable opportunistic use of TV band white spaces, the idea of taking advantage of knowledge of position information about the primary users to optimize cognitive radio network operations and in particular route selection is appealing beyond the specific white spaces application scenario.

Several position-based routing protocols have been proposed in the past, that use position information either to reduce overhead during route search procedures ([1], [2], [3]), or to select the next hop in data packet forwarding ([4], [5], [6]). More recently, the problem of position-based routing was addressed in the context of indoor application scenarios, where GPS positioning is not available, and terminals have to rely on indoor location services for obtaining position information [7], [8], [9], [10].

The above solutions rely on two key assumptions:

1) omnidirectional antennas are deployed at each terminal;

2) each terminal is at all times aware of its own position, and location services are only used by a device to obtain information about the position of other terminals.

This work addresses the concept of position-based routing in the context of underlay cognitive wireless communications, and takes a more general approach by removing the two above assumptions, aiming at a solution that a) takes advantage of position information in order to adapt the antenna pattern, so to maximize emissions in preferred directions, and b) operates under the assumption that position information is built based on the cooperation between terminals, with no predefined information being available.

The solution proposed in this work towards the achievement of the above goal is to equip each cognitive device with an array of antenna elements, capable of dynamically steering the antenna beam by means of beamforming, while providing a way of collecting position information by means of cooperation between devices implementing a Direction Of Arrival (DOA) positioning technique.

The adoption of DOA-based position information and beamforming in routing for cognitive wireless networks opens new interesting opportunities:

- to increase the coexistence capabilities of the cognitive network by avoiding positions interested by the presence of primary/coexisting systems on both a network-wide scale (by selecting routes far away from interested locations) and a local scale (by steering the antenna beam so to place nulls in the direction of potential interference victims); 


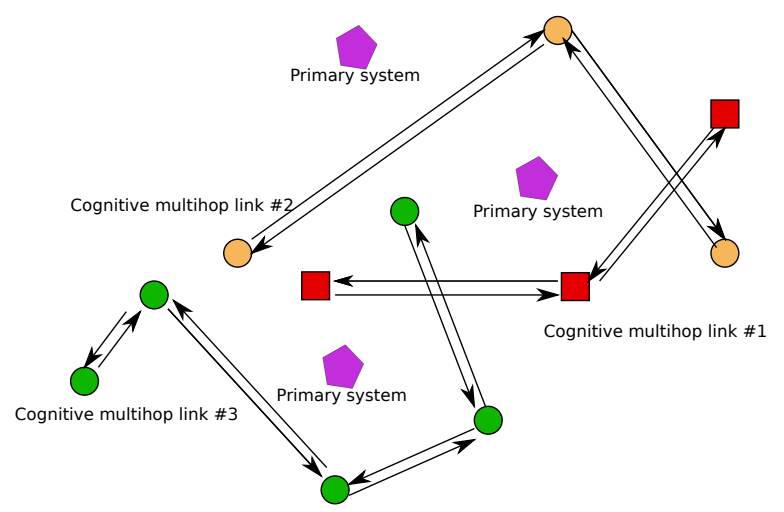

Fig. 1. Scenario considered in this work (Circles and squares: secondary nodes; pentagons: primary systems).

- to maximize the efficiency in the use of available power by placing the maximum of the beam in the direction of the intended receiver (next hop along the path).

The deployment of the proposed solution also poses significant research challenges:

- to define algorithms and protocols for building the position information through local cooperation and for guaranteeing the consistency and coherence of such information on a network-wide scale;

- to integrate the relative position information provided by the DOA technique with absolute position information about primary systems locations provided by external databases;

- to cope with the impact of positioning errors, affecting, in turn, the efficiency of the beamforming.

This paper constitutes a first step in addressing the above issues. The work focuses on a network scenario characterized by a few concurrent multihop traffic streams in the cognitive network, being deployed in the presence of primary victim receivers in known location, as shown in Figure 1.

The paper is organized as follows. Section II introduces the location-based routing protocols considered in this work. Section III introduces the algorithms adopted for the estimation of the direction of arrivals of the signals and the beamforming, and Section IV illustrates the proposed routing strategy. Section $\mathrm{V}$ presents a performance evaluation by comparing the proposed solution applied to two different routing protocols identified in Section II to a standard non location-enabled solution. Finally, Section VI draws conclusions.

\section{LOCATION-BASED ROUTING PROTOCOLS}

As already noted in Section I, solutions for introducing position information in routing can be divided in two categories, that is source routing protocols, that use position information during the route search procedure, and simple packet forwarding protocols that use position information to select the next hop in packet forwarding [10]. In the present Section a protocol for each of the two categories will be considered. The two protocols are described below.

As a representative of the first category, the Location-Aided
Routing (LAR) protocol will be adopted [1]. The LAR protocol is a typical on-demand routing protocol. In order to find a route between source and destination terminal, it relies on a flooding-based Route Discovery procedure. Source S starts the procedure by broadcasting a Route Request packet to all its neighbours. The packet contains Source and Destination IDs, and a sequence number that uniquely identifies the connection request. Each terminal I that receives a Route Request packet checks two different conditions:

- I is the intended destination of the packet: if yes, the packet is processed without forwarding;

- another packet characterized by the same Source, Destination and sequence number has been already received: if yes the packet is discarded without forwarding.

If none of the conditions is satisfied the packet is updated, by including the ID of I in the path recorded in the packet, and forwarded to its neighbours. When a Route Request packet reaches the desitination $\mathrm{D}$, a Route Reply procedure is executed where D sends a reply packet on a backward path obtained by reverting the forward path; following the reception of the Route Reply packet S starts sending data packets to D. Both Route Discovery and Route Reply procedures may fail for different reasons, such as lack of network connectivity between $\mathrm{S}$ and $\mathrm{D}$ or communication errors. In order to manage such situations a timeout is set by $\mathrm{S}$ when a Route Discovery procedure is activated. If the timeout expires before a Route Reply packet is received, a new Route Discovery is started, characterized by a different sequence number.

Compared to traditional source routing protocols, such as Dynamic Source Routing (DSR), the LAR protocol optimizes the Route Discovery procedure by taking advantage of position information. The position information required by LAR consists in source position and destination position. Additionally, an estimation of maximum terminal speed is required in case of mobility. Such information is exploited during the Route Discovery procedure in following way.

Suppose that a terminal S starts a Route Discovery procedure to destination $\mathrm{D}$ at time $t=t_{1}$, and that the last information update regarding the position of $\mathrm{D}\left(x_{D}\left(t_{0}\right), y_{D}\left(t_{0}\right)\right)$ was received by $\mathrm{S}$ in $t=t_{0}$. Based on the estimation of the maximum speed $v$ of terminal $\mathrm{D}, \mathrm{S}$ can evaluate the maximum distance travelled by D since the last location update. Such a distance is given by $d_{M A X}=v \cdot\left(t_{1}-t_{0}\right)$. As a consequence, the current position occupied by $\mathrm{D}$ lies in a circular region of radius $d_{M A X}$ centered on $\left(x_{D}\left(t_{0}\right), y_{D}\left(t_{0}\right)\right)$, referred to as the Expected zone (Figure 2). The Expected zone indicates which zone of the network should be reached by Route Request packets. The idea behind LAR is to exploit this information to reduce the amount of Route Request packets flooding through the network, by allowing forwarding of packets generated by the source only in the direction of the Expected zone containing the destination. The region of the network in which forwarding is allowed is referred to as Request zone. An intermediate terminal is allowed to forward a Route Request packet only if it lies within the Request zone defined by the 


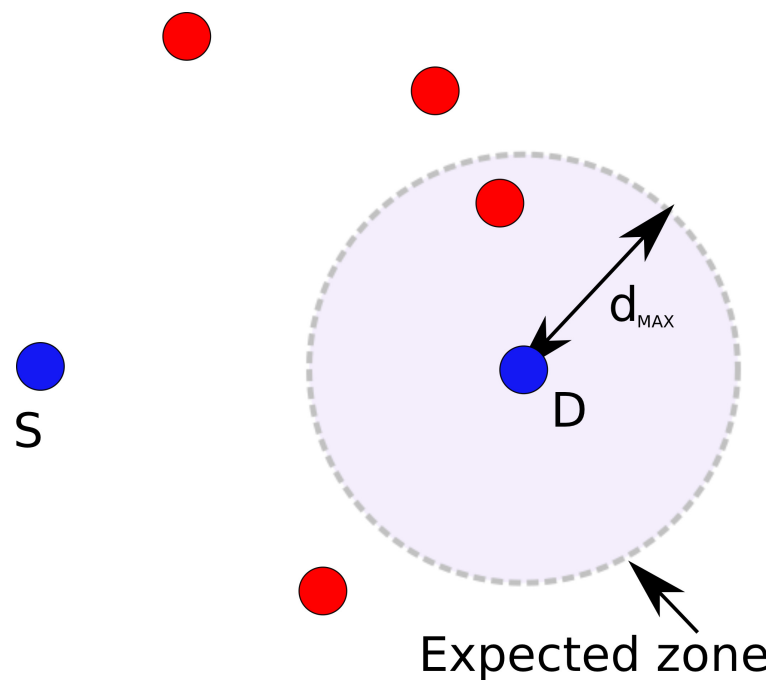

Fig. 2. Definition of Expected zone in the LAR protocol.

source of the connection request.

The fundamental property of the Request zone is that it contains both the Expected zone and the position of the source terminal S. Provided that this condition is met, different definitions are possible for such a zone. The definition of the Request zone is the result of a trade off between two opposite phenomena:

- A smaller Request zone leads to a lower routing overhead, as a lower number of terminals is allowed to forward packets;

- A larger Request zone increases the probability of finding a path to the destination, in particular in the case of sparse networks.

The protocol considered for the second category is the Greedy Perimeter Stateless Routing (GPSR) protocol. GPSR uses location information to reduce protocol overhead and get a good scalability as both terminal mobility and network size increase [4].

GPSR adopts positional information as the key metric in packet forwarding, using a simple "greedy" forwarding strategy:

1) Each packet is marked by the source terminal with the information about location of the destination;

2) Each intermediate node forwards the packet to the neighbouring node at minimum distance from the location of the destination recorded in the packet itself.

Note that such a strategy by itself does not guarantee that a path between source and destination is always detected, as situations may occur in which a terminal is closer to the destination than any of its neighbours, thus achieving a local minimum in the distance from the destination. In these cases in [4] it is proposed to switch from a greedy forwarding strategy to a perimeter forwarding strategy based on planar graphs theory, in which a terminal is allowed to forward the packet to a neighbour which is farther than itself from the destination, in order to solve the stall caused by greedy forwarding.
Several other works focus on the issue of recovering from local minima, and mostly rely on planar graph theory as well [11], [12]. In the present work the stall is solved by following a simpler but more robust approach, based on the fallback solution of reverting to flooding, so to send the packet to all neighbours and eventually overcome the stall situation.

\section{Direction Of ARrival estimation AND BEAMFORMING}

The problem of determining the direction of arrival of a signal by means of an array of antenna elements was studied extensively by the research community. A well known approach relies on subspace decomposition of a covariance matrix built from the signal received on each antenna array element from the targets: this was the basis for the definition of the MUSIC algorithm [13], that allows to build a function of the angle of arrival, referred to as MUSIC pseudospectrum, that shows peaks at angles corresponding to direction of arrival of signals emitted by other devices.

Moving from the knowledge of DOA of a signal at two different receivers, the position of the terminal emitting the signal can be determined, provided that the position of the two receivers is known as well. In the ideal case of error-free DOA estimation, the determination of the position of the emitter is a simple geometrical problem. In the more realistic case of DOA estimations affected by noise, both iterative and non iterative approaches have been proposed to solve the problem [14], [15].

The array of antenna elements can be used not only to determine the direction of arrival of signals, but also to avoid emitting towards specific directions, by adopting a technique known as beamforming; the coexistence capabilities of a secondary network can be significantly improved by imposing nulls on directions leading to the positions occupied by primary systems. The beamforming technique used in this work is more commonly referred to as Orthogonal Transmit Beamforming (OTBF), where the transmitted signals are made orthogonal to each other, so that co-channel interference is completely suppressed, without any further processing at the receiver end. The main goal of the beamforming technique is satisfy the signal-to-interference-plus-noise ratios (SINR) of the secondary users while keeping the interference to the primary users below a certain threshold. Instead of instantaneous channel gains, the second order statistics of the channel are used at the secondary transmitters. The direction of the beam pattern is determined by utilizing the DOA information. More details can be found in [16].

\section{LOCATION-BASED ROUTING BASED ON DOA AND BEAMFORMING}

The solution proposed in this work combines the protocols for location-based routing described in Section II with the DOA and beamforming techniques introduced in Section III, focusing on the specific case of an underlay secondary network. Note that the assumption of an underlay physical 


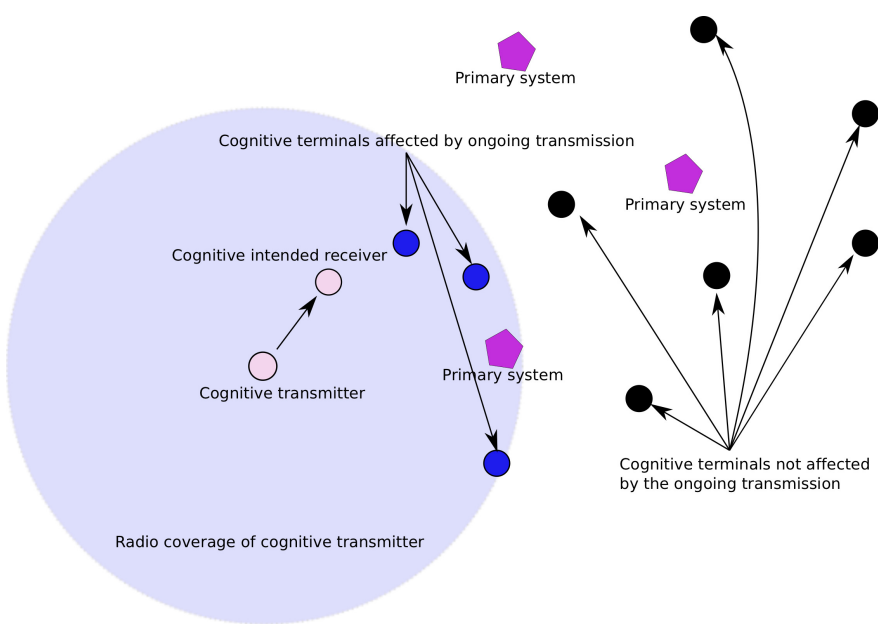

Fig. 3. Example of application scenario of beamforming to routing in the scenario considered in the present work (Light circles: secondary nodes involved in the packet exchange; dark circles: secondary nodes affected by the packet exchange; black circles: secondary nodes not affected by the packet exchange; pentagons: primary systems).

layer decouples the problem of best route selection from the issue of hop-by-hop channel selection typical of interweave cognitive networks, as all devices in the secondary network are assumed to share the same wideband underlay channel encompassing the frequencies used by the primary systems.

The goal is to take advantage of the position information obtained by DOA to select the best route along the network, while at the same time reducing the impact of each hop in the route on potential victim receivers by introducing beamforming. It should be noted that in this work the beamforming is adopted by each terminal transmitting a packet to reduce the interference generated to both primary receivers and other secondary receivers that are not the intended recipients of the packet. With reference to the scenario introduced in Figure 1, an example of application of beamforming is shown in Figure 3 . Figure 3 highlights that secondary nodes should be protected from the effects of an ongoing transmission in the same way as primary receivers interested by the same transmission. This can be achieved by redefining the problem originally addressed in [16] so to consider both primary systems and secondary nodes not involved in the communication as victim receivers, and thus optimize the emission beam in order to guarantee a maximum level of interference towards such devices. Such optimization can only be performed when a terminal is sending unicast packets (although multicast transmissions could be handled as well, adopting an approach closer to what is proposed in [16]), after the conclusion of broadcast transmissions, usually required for setting up the route by choosing the endto-end path. The proposed approach can be thus organized in two phases:

1) select the end-to-end path according to the procedure defined in the routing protocol, without introducing beamforming;

2) apply beamforming as discussed above to the data packet transmissions taking place along the route identified in Phase 1.

Note that Phase 1 is heavily depending on the selected routing protocol. In some cases, as in the LAR routing protocol presented in Section II, such step is a key aspect in the routing process; in other cases, as in the GPSR routing protocol also introduced in Section II, Phase 1 is completely absent, as the protocol does not perform any route search before transmitting the data packets. In addition, it is worth noting that, independently from the adoption of beamforming, both steps can benefit from the introduction of position information provided by the DOA technique. Note that the selection of a routing metric is a key step in the definition of Phase 1 procedures.

As a final comment, in the present version of the work routing metric is kept as simple as possible: in the case of LAR and DSR, the destination D starts the Route Reply procedure as soon as the first Route Request packet is received; in the case of GPSR distance to destination is the only parameter taken into account on the selection of next hop. The adoption of more complex routing metrics, taking into account coexistence and communications requirements, as investigated for example in [17], [18], [19], will be explored in a future extension of the work.

\section{PERformance EVAluation}

The performance of the solution proposed in Section IV was evaluated by computer simulations based on the combination of MatLab (for the solution of the optimization process connected to the selection of the beamforming weights) and the discrete event simulator OMNeT++ [20] for the implementation of the routing protocols. Simulations were carried out in a scenario characterized by the presence of $N=10$ underlay secondary still nodes adopting an UWB physical layer, coexisting with a primary receiver over an area of dimensions equal to $100 \times 100$ square meters. Simulations were performed as follows.

1) Network topologies were generated with MATLAB. In each topology the beamforming problem was solved for all possible transmitter-receiver pairs, determining for all other terminals the Interference Reduction Factor (IRF), defined as the ratio between the interference they would have experienced without beamforming and the actual experienced interference. This led to the creation of a $N \times N \times N$ tridimensional matrix named $I R F$, where $\operatorname{IRF}(i, j, k)$ contained the Interference Reduction Factor experienced by terminal $k$ when terminal $i$ is transmitting to terminal $j$.

2) Connection requests were generated in $\mathrm{OMNeT}++$, allowing a subset of nodes (nodes 0,1 and 2) to generate connection requests, and end-to-end routes were identified according to the selected routing protocol: LAR+beamforming (LAR-BF), GPSR+beamforming (GPSR-BF) or a DSR-like protocol, not using information on position of terminals. For each connection, after the selection of the route, beamforming was introduced 


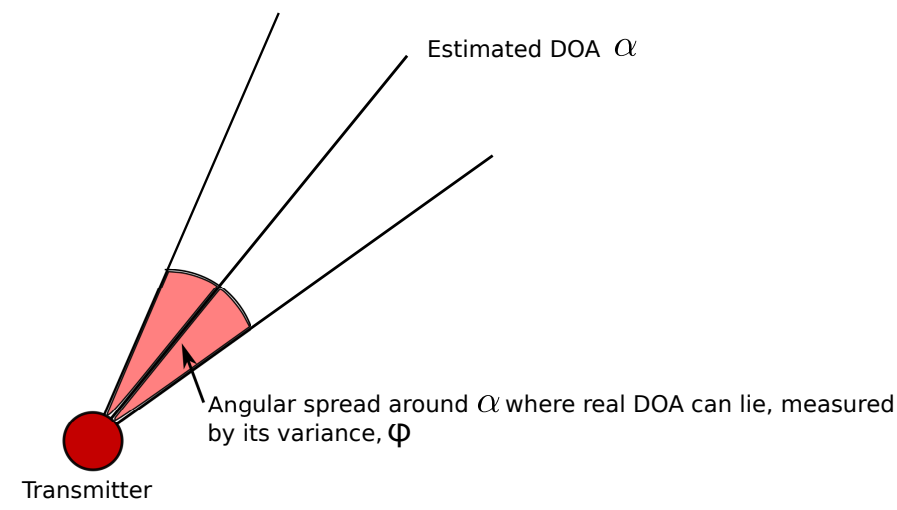

Fig. 4. Definition of error in DOA estimation. The line departing from the transmitter identifies the nominal DOA $\alpha$ estimated by the transmitter itself for a potential receiver, and the angular spread around the line represents the actual range of DOAs where the real DOA can lie. The spread is characterized by its variance $\phi$.

TABLE I

MAIN SIMULATION SETTINGS

\begin{tabular}{cc}
\hline Parameter & Value \\
\hline Connection bit rate & $10 \mathrm{~kb} / \mathrm{s}$ \\
\hline Transmission rate & $966 \mathrm{~kb} / \mathrm{s}$ \\
\hline Power & $3.65 \mu \mathrm{W}$ \\
\hline DATA packet length & 1224 bits $(+64$ bits for Sync trailer) \\
\hline Secondary devices physical layer settings & Impulse Radio UWB with $500 \mathrm{MHz}$ bandwidth \\
\hline DOA angular spread variance & $0,2,4 \mathrm{rad}^{2}$ \\
\hline Maximum uniform positioning error & $0,2,4 \mathrm{~m}$ \\
\hline Number of antenna elements in each secondary device & 8 \\
\hline
\end{tabular}

in the DATA packets routing process by importing the values of the IRF function corresponding to the topology being used.

Simulations took into account the accuracy in DOA estimation and, in turn, in position estimation. In accordance to the approach proposed in [16], the accuracy in DOA estimation was measured by the variance of the angular spread of the real DOA of the signal by a given user around the nominal estimated DOA, as shown in Figure 4.

Error in position estimation was modeled as a uniform variable distributed between 0 and the numerical value of the variance of the angular spread. The introduction of values of the positioning error analytically derived from the DOA estimation error is left for future work.

Main simulation parameters are presented in Table I.

Figure 5 presents the end-to-end throughput, defined as the ratio between received packets and transmitted packets, for LAR-BF, GPSR-BF and DSR for three different cases: no DOA/positioning error, low DOA/positioning error and high DOA/positioning error. The results shown in Figure 5 highlight the increase in throughput due to the reduction of Packet Error Rate guaranteed by the introduction of beamforming, in particular in the case of LAR-BF. Beamforming leads in fact to a reduction of interference, as shown in Figure 6, presenting the IRF as a function of the DOA estimation error measured by the variance of the angular spread. Figure 7, presenting the number of transmitted Route Request packets as a function

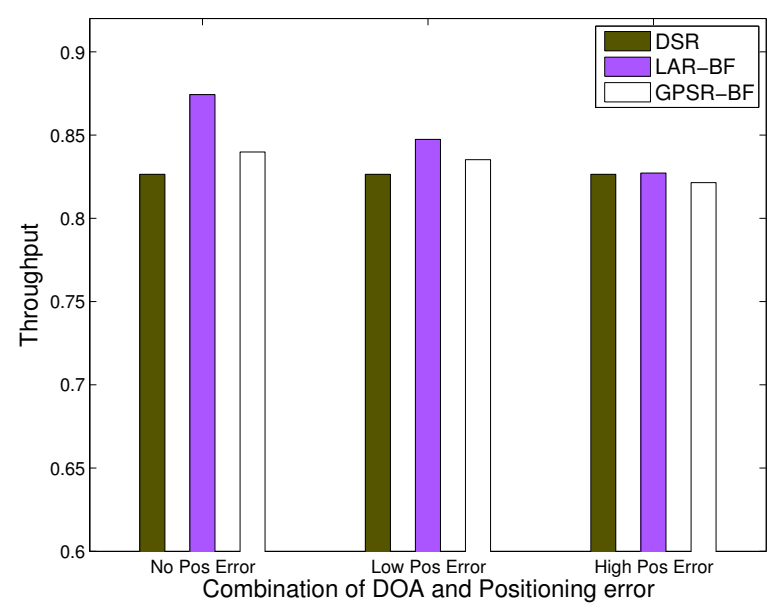

Fig. 5. End-to-end throughput, defined as the ratio between received packets and transmitted packets, for LAR-BF, GPSR-BF and DSR in three different scenarios (No Pos Error: no DOA error, no positioning error; Low Pos Error: Variance of angular spread $=2 \mathrm{rad}^{2}$, uniform positioning error with $\max =2$ $m$; High Pos Error: Variance of angular spread $=4 \mathrm{rad}^{2}$, uniform positioning error with $\max =4 \mathrm{~m}$ ). Note that in the case of DSR the result is the same for all the three cases as the protocol does not rely on position information.

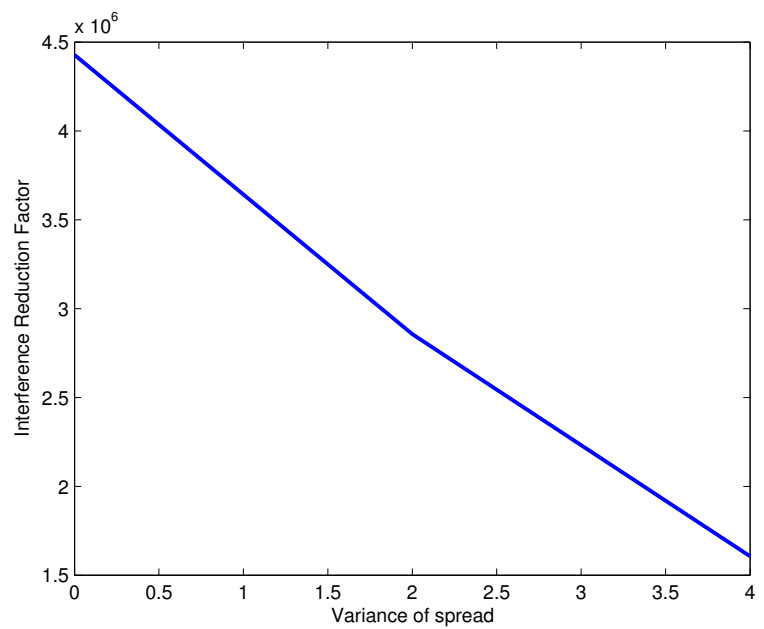

Fig. 6. Interference Reduction Factor as a function of the variance of the angular spread.

of time, shows that the introduction of position information in routing also contributes to the increase of the network performance guaranteed by LAR-BF, as it leads to a significant reduction in the number of control packets during the route search phase further reducing the impact of packet collisions and allowing for energy savings. GPSR is not reported here as it does not foresee a route discovery phase.

As a general comment to simulation results, it can be noted that the advantage provided by the introduction of beamforming and position information is interesting, but not astonishing. It is worth observing, however, that the increase in performance can be expected to become more and more relevant as network size and density increase, as the MUI and 


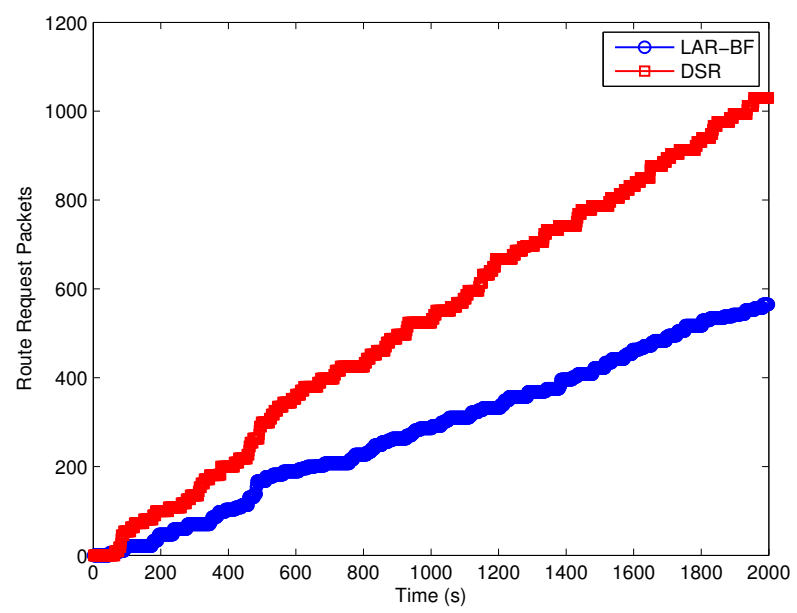

Fig. 7. Number of Route Request Packets as a function of time for LAR-BF vs. DSR.

control traffic reduction become more and more pronounced. Performance analysis in scenarios characterized by larger number of terminals is indeed planned as part of future work. On the other hand, a complete assessment of the effectiveness of the proposed solution will also require to take into account the additional exchanges required to collect and update the DOA and position information.

\section{CONCLUSION}

This work focused on the definition of a new routing strategy for cognitive wireless networks, based on the combination of a position-based routing protocol with a beamforming technique aiming at maximizing the SNR at the intended receiver while minimizing the interference caused at other affected receivers, either belonging at the secondary network or to primary networks coexisting in the same area. Both position-based routing and beamforming rely on position estimation built on Direction Of Arrival estimation, enabled by the adoption of an uniform array of antenna elements at each transmitter. The work introduced two position-based routing protocols, LAR and GPSR, considered as suitable for integration with DOA and beamforming towards the creation of the routing strategy. Next, DOA and beamforming were briefly introduced, and the proposed strategy was presented and evaluated by means of computer simulation. Results highlight the potential advantage over traditional, non-positionaware routing strategies, that can be achieved by the proposed strategy, and suggest the need of further studies to quantify such advantage. Future work will focus on taking into account additional implementation aspects, such as the overhead for building position information from DOA estimates through cooperative distributed positioning, as well as on introducing advanced routing metrics in the proposed strategy.

\section{ACKNOWLEDGMENT}

This paper has been supported by the ICT-ACROPOLIS Network of Excellence, FP7 project n. 257626 and by COST
Action IC0902 "Cognitive Radio and Networking for Cooperative Coexistence of Heterogeneous Wireless Networks".

\section{REFERENCES}

[1] Y. B. Ko and N. H. Vaidya, "Location aided routing in mobile ad hoc networks," in ACM International Conference on Mobile Computing and Networking (Mobicom), October 1998, pp. 66-75.

[2] D. Kim, Y. Choi, and C. K. Toh, "Location-aware long lived-route selection in wireless ad hoc networks," in IEEE Vehicular Technology Conference, vol. 4, 2000, pp. 1914-1919.

[3] S. Basagni, I. Chlamtac, and V. R. Syrotiuk, "Dynamic source routing for ad-hoc networks using the global positioning system," in IEEE Wireless Communications and Networking Conference, September 1999, pp. 301305.

[4] B. Karp and H. T. Kung, "Gpsr: Greedy perimeter stateless routing for wireless networks," in ACM International Conference on Mobile Computing and Networking (Mobicom), 2000, pp. 243-254.

[5] S. Basagni, I. Chlamtac, V. R. Syrotiuk, and B. A. Woodward, "A distance routing effect algorithm for mobility (DREAM)," in ACM/IEEE International Conference on Mobile Computing and Networking (Mobicom), 1998, pp. 25-30.

[6] A. M. Akhtar, M. R. Nakhai, and A. H. Aghvami, "Adaptive power aware routing in wireless mesh networks," in IEEE Vehicular Technology Conference, vol. 1, May 15-18 2011, pp. 1-5.

[7] H. Cheng and J. Cao, "A design framework and taxonomy for hybrid routing protocols in mobile ad hoc networks," IEEE Communications Surveys \& Tutorials, vol. 10, no. 3, pp. 62-73, 2008.

[8] E. Kuiper and S. Nadjm-Tehrani, "Geographical Routing With Location Service in Intermittently Connected MANETs," IEEE Transactions on Vehicular Technology, vol. 60, no. 2, pp. 592-604, February 2011.

[9] T. Melodia, D. Pompili, and I. Akyildiz, "On the interdependence of distributed topology control and geographical routing in ad hoc and sensor networks," IEEE Journal on Selected Areas in Communications, vol. 23, no. 3, pp. 520-532, March 2005.

[10] I. Stojmenovic, "Position-based routing in ad hoc networks," IEEE Communications Magazine, vol. 40, no. 7, pp. 128-134, July 2002.

[11] I. Bose, P. Morin, and J. Urrutia, "Routing with Guaranteed Delivery in Ad Hoc Wireless Networks," in Proceedings of Third ACM International Workshop on Discrete Algorithms and Methods for Mobile Computing and Communications, 1999, pp. 48-55.

[12] F. Kuhn, W. R., and Z. A., "Worst-Case Optimal and Average-Case Efficient Geometric Ad-Hoc Routing," in Proceedings of ACM MobiCom, 2003, pp. 267-278.

[13] R. O. Schmidt, "Multiple emitter location and signal parameter estimation," IEEE Transactions on antennas and propagation, vol. AP-34, no. 3, pp. 276-280, 1986.

[14] D. J. Torrieri, "Statistical theory of passive location systems," IEEE Trans. on AES, vol. AES-20, no. 2, pp. 183-198, March 1984.

[15] A. Pagès-Zamora, J. Vidal, and D. Brooks, "Closed-form solution for positioning based on angle of arrival measurements," in IEEE International Symposium on Personal, Indoor and Mobile Radio Communications, September 2002, pp. $1522-1526$.

[16] A. M. Akhtar, O. Holland, T. A. Le, M. R. Nakhai, and A. A. H., "Cooperative cognitive radio beamforming in the presence of location errors," in IEEE Vehicular Technology Conference, 2011, pp. 1-5.

[17] K. Chowdhury and I. Akyildiz, "CRP: A Routing Protocol for Cognitive Radio Ad Hoc Networks," IEEE Journal on Selected Areas in Communications, vol. 29, no. 4, pp. 794-804, April 2011.

[18] M.-G. Di Benedetto and L. De Nardis, "Cognitive routing models in UWB networks," in IEEE International Conference on Cognitive Radio Oriented Wireless Networks and Communications (CROWNCOM), May 2008.

[19] P. Baldi, L. De Nardis, and M. G. Di Benedetto, "Modeling and Optimization of UWB Communication Networks through a flexible cost function," IEEE Journal on Selected Areas in Communications, vol. 20, no. 9, pp. 1733-1744, December 2002.

[20] A. Varga, "OMNeT++," IEEE Network Interactive, vol. 16, no. 4, July 2002. 